\title{
The Dominance of Ossicular Route in Sound Transmission
}

\author{
Mohd Normani Zakaria ${ }^{1}$ - Aw Cheu Lih ${ }^{1} \cdot$ Noor Alaudin Abdul Wahab² \\ ${ }^{1}$ Department of Audiology, School of Health Sciences, Universiti Sains Malaysia, Kubang Kerian; \\ ${ }^{2}$ Department of Audiology, School of Rehabilitation Sciences, Faculty of Health Sciences, National University of Malaysia, \\ Kuala Lumpur, Malaysia
}

\section{To the editor:}

We read the article of Park et al. [1] with great interest. The finding obtained from this study further supports the notion that the location of tympanic membrane (TM) perforation has no influence on the degree of hearing loss, which contradicts the popular belief (i.e., the posterior quadrant of TM produces more hearing loss than the anterior part of TM). Furthermore, the study has utilized a novel method to evaluate middle ear and mastoid pneumatization volumes which are important indicators in determining the degree of hearing loss.

Early studies in 1970s revealed that the location of TM perforation had influence on the severity of hearing loss. That is, due to the direct sound exposure to oval and round windows (causing phase-cancellation effect), perforation on the posterior part of TM produced more hearing loss than the anterior portion of TM [2]. However, more recent studies have shown that the perforation location on TM has no relation to the degree of hearing loss $[3,4]$. Furthermore, the discrepancy in result could be possibly because the middle ear volume was not taken into consideration in the earlier studies [4].

In the study of Park et al. [1], the majority of subjects tested had small perforation sizes $(<30 \%)$ and the mean air-bone gaps were less than 20-dB hearing level (HL). Such small air-bone gaps in TM perforation cases were also observed in the study of Mehta et al. [4]. In this regard, the ossicular route was still dominant in transmitting sounds and hence, no relation between the site of TM perforation and hearing level would be expected (i.e.,

\footnotetext{
- Received November 10, 2015

Accepted November 19, 2015

- Corresponding author: Mohd Normani Zakaria

Department of Audiology, School of Health Sciences, Universiti Sains

Malaysia, 16150 Kubang Kerian, Kelantan, Malaysia

Tel: +60-9-7677691, Fax: +60-9-7677515

E-mail: mdnorman@usm.my
}

the phase-cancellation effect was not relevant as the sound transmission via the acoustic route was negligible). When the TM perforation size is big enough and the hearing level exceeds $40-\mathrm{dB}$ HL, the acoustic route would become dominant [5]. Perhaps this point is worthy of consideration to further educate the readers.

Experimental studies on cadavers have found no relation between the site of TM perforation and the hearing level, even when the sound transmission is dominated by the acoustic route [3,5]. On the other hand, evidence gathered from clinical studies is scarce. That is, in cases where TM perforation sizes were big, significantly bigger hearing losses were still noted for the posterior location of TM [6]. Accordingly, future well-controlled clinical studies are warranted before a concrete conclusion can be made in regard to this controversial issue.

\section{CONFLICT OF INTEREST}

No potential conflict of interest relevant to this article was reported.

\section{REFERENCES}

1. Park H, Hong SN, Kim HS, Han JJ, Chung J, Seo MW, et al. Determinants of conductive hearing loss in tympanic membrane perforation. Clin Exp Otorhinolaryngol. 2015 Jun;8(2):92-6.

2. Anthony WP, Harrison CW.Tympanic membrane perforation: effect on audiogram. Arch Otolaryngol. 1972 Jun;95(6):506-10.

3. Voss SE, Rosowski JJ, Merchant SN, Peake WT. How do tympanicmembrane perforations affect human middle-ear sound transmission? Acta Otolaryngol. 2001 Jan;121(2):169-73.

4. Mehta RP, Rosowski JJ, Voss SE, O'Neil E, Merchant SN. Determinants of hearing loss in perforations of the tympanic membrane. Otol Neurotol. 2006 Feb;27(2):136-43.

5. Voss SE, Rosowski JJ, Merchant SN, PeakeWT. Non-ossicular signal

Copyright @ 2016 by Korean Society of Otorhinolaryngology-Head and Neck Surgery.

This is an open-access article distributed under the terms of the Creative Commons Attribution Non-Commercial License (http://creativecommons.org/licenses/by-nc/4.0)

which permits unrestricted non-commercial use, distribution, and reproduction in any medium, provided the original work is properly cited. 
transmission in human middle ears: experimental assessment of the "acoustic route" with perforated tympanic membranes. J Acoust SocAm. 2007 Oct;122(4):2135-53.
6. Bhusal CL, Guragain RP, Shrivastav RP. Frequency dependence of hearing loss with perforations. JNMA J Nepal Med Assoc. 2007 OctDec;46(168):180-4. 